\title{
Rational approximant structures to decagonal quasicrystals
}

\author{
S. Ranganathan*, Anandh Subramaniam, K. Ramakrishnan \\ Department of Metallurgy, Indian Institute of Science, Bangalore 560012, India
}

\begin{abstract}
We have shown earlier that the decagonal quasicrystalline phase can be derived by the twinning of the icosahedral cluster about the five-fold axis by $36^{\circ}$. It is shown here that in a similar fashion, the rational approximant structures (RAS) to the decagonal quasicrystal can be constructed by the twinning of RAS to the icosahedral quasicrystalline phase. The twinning of the Mackay (cubic) type RAS leads to the Taylor $(q 1 / p 1, q 1 / p 1)$ phases, while the twinning of the orthorhombic Little phase leads to the Robinson $(q 1 / p 1, q 2 / p 2)$ approximants to the decagonal quasicrystal. With increasing order of $q 1 / p 1$ or $q 2 / p 2$, we approach the digonal quasicrystal with one-dimensional quasiperiodicity. (C) 2001 Published by Elsevier Science B.V.
\end{abstract}

Keywords: Rational approximant structures (RAS); Quasicrystalline phase; Taylor and the Robinson phases

\section{Introduction}

Orthorhombic approximants have been identified for the decagonal quasicrystal. The periodic axis of the decagonal phase is inherited as one of the major axes of the approximant phase, usually denoted as the $b$ axis. The other two axes are parallel to the two two-fold directions (2D and $2 \mathrm{P}$ ) of the decagonal phase. These axes are denoted as $q / p$. Zhang and Kuo [1] proposed that the "decagonal approximants" can be represented as $(q 1 / p 1, q 2 / p 2)$, corresponding to the $a$ and $c$ axes. The distance of intense spots along one two-fold direction in the 10 -fold pattern is a little longer than the other. Therefore, the $a$ and $c$ axes of the decagonal approximants are not equal.

There are two types of orthorhombic approximants to the decagonal phase. They have been termed the Taylor and the Robinson phases. The Taylor type is the one in which the $a$ and $c$ axes are approximated by the same $q / p$ ratio and for the Robinson type, the $a$ and $c$ axes are approximated by different $q / p$ ratio. The Taylor phase $\mathrm{Al}_{3} \mathrm{Mn}$ (space group: Pnma; $a=1.259 \mathrm{~nm} ; b=1.242 \mathrm{~nm} ; c=1.48 \mathrm{~nm}$ ) was first found in Al-Mn binary system [2,3]. The Taylor phase has been seen in several alloy systems, namely, high temperature $\mathrm{Al}_{11}(\mathrm{Mn}, \mathrm{Pd})_{4}$ (or $\mathrm{Al}_{3}(\mathrm{Mn}, \mathrm{Pd})$ ) [4], Y-AlMnCu [5,6], T-Al ${ }_{71} \mathrm{Mn}_{23} \mathrm{Pd}_{6}$ [7-9]. A structural model for $\mathrm{Al}_{3} \mathrm{Mn}$ phase has been proposed by Hiraga et al. [10]. The structure consists of a column of small pentagons surrounded by larger pentagons and decagons giving rise to a columnar

\footnotetext{
* Corresponding author. Tel.: +91-80-3092558; fax: +91-80-3312991 E-mail address: rangu@metalrg.iisc.ernet.in (S. Ranganathan).
}

structure. It should be noted that the icosahedra building the pentagonal columns occur in two orientations. This is an important feature of the decagonal quasicrystal and its approximants.

The Robinson phase $\mathrm{Al}_{60} \mathrm{Mn}_{11} \mathrm{Ni}_{4}$ [11-13], is an orthorhombic ( $a=0.755 \mathrm{~nm} ; b=1.25 \mathrm{~nm} ; c=2.38 \mathrm{~nm}$; space group: $\mathrm{Cmcm}$ ) approximant to the decagonal quasicrystal. Robinson [12] noted the pentagonal coordination and a layering in the structure of $\mathrm{Al}_{60} \mathrm{Mn}_{11} \mathrm{Ni}_{4}$ orthorhombic phase. There are two phases with structures similar to this Robinson phase in Al-Mn-Cu [14] and Al-Mn-Zn alloys [13]. The Robinson phase is reported to coexist with the Taylor phase in Al-Mn [15], Al-Mn-Cu [16,17] and Al-Mn-Pd [9]. There are several examples reported in the literature (Table 1).

Audier et al. [9] have calculated the relationship between the lattice parameters of the Robinson and the Taylor phase and the decagonal quasicrystal. For the Robinson phase, the lattice parameter relationship with the decagonal quasicrystal is

$$
\begin{aligned}
& a_{\mathrm{R}}=a_{5 \mathrm{D}} \sqrt{\tau+2} \sqrt{\frac{2}{5}} \\
& b_{\mathrm{R}}=c_{\mathrm{D}} \\
& c_{\mathrm{R}}=a_{5 \mathrm{D}}(3 \tau+1) \sqrt{\frac{2}{5}}
\end{aligned}
$$

where, $a_{5 \mathrm{D}}$ is the hyper-cell parameter related to the decagonal quasicrystal $\left(a_{5 \mathrm{D}}=0.6451 \mathrm{~nm}\right)$. For the Taylor phase, the lattice parameter relationship with the decagonal quasicrystal is 
Table 1

Examples of Robinson and Taylor approximants to the decagonal quasicrystal

\begin{tabular}{llllll}
\hline Approximant phases & $\begin{array}{l}\text { Order of the } \\
\text { approximant }\end{array}$ & \multicolumn{3}{l}{ Lattice parameter } \\
\cline { 4 - 6 } & & $a(\mathrm{~nm})$ & $b(\mathrm{~nm})$ & $c(\mathrm{~nm})$ \\
\hline Taylor approximants & & & & \\
$\quad \mathrm{Al}_{3} \mathrm{Mn}$ & $(3 / 2,3 / 2)$ & 1.259 & 1.242 & 1.48 \\
$\mathrm{Al}$ & $\mathrm{P} \mathrm{Pd}_{5} \mathrm{Mn}_{25}$ & $(3 / 2,3 / 2)$ & 1.251 & 1.243 & 1.483 \\
$\mathrm{Al}-\mathrm{Co}-\mathrm{Cu}$ & $(5 / 3,5 / 3)$ & 1.97 & 0.4 & 2.33 \\
$\mathrm{Robinson}$ approximants & & & & \\
$\mathrm{Al}_{60} \mathrm{Mn}_{11} \mathrm{Ni}_{4}$ & $(2 / 1,5 / 3)$ & 0.755 & 1.25 & 2.38 \\
$\mathrm{Al}_{20} \mathrm{Mn}_{3} \mathrm{Cu}_{2}$ & $(2 / 1,5 / 3)$ & 0.772 & 1.25 & 2.42 \\
$\mathrm{Al}_{11} \mathrm{Mn}_{3} \mathrm{Zn}_{2}$ & $(2 / 1,5 / 3)$ & 0.778 & 1.26 & 2.38 \\
\hline
\end{tabular}

$a_{\mathrm{T}}=a_{5 \mathrm{D}} \tau \sqrt{\tau+2} \sqrt{\frac{2}{5}}$

$b_{\mathrm{T}}=c_{\mathrm{D}}$

$c_{\mathrm{T}}=2 a_{5 \mathrm{D}}(\tau+2) \sqrt{\frac{2}{5}}$

A relationship between the lattice parameters of the Robinson phase and the Taylor phase can be derived as

$a_{\mathrm{R}}=a_{\mathrm{T}} \frac{1}{2 \tau}$

$c_{\mathrm{R}}=c_{\mathrm{T}} \frac{3 \tau+1}{2(\tau+2)}$

$b_{\mathrm{R}}=b_{\mathrm{T}}$

The orientation relationship between the decagonal quasicrystal, the Robinson phase and the Taylor phase is $\left(\begin{array}{lll}0 & 1 & 0\end{array}\right)_{\mathrm{D}}\left\|\left(\begin{array}{lll}0 & 1 & 0\end{array}\right)_{\mathrm{T}}\right\|\left(\begin{array}{lll}0 & 1 & 0\end{array}\right)_{\mathrm{R}}$.

Both these phases have their $b$-axis parallel to the 10 -fold axis of the decagonal phase and the two-fold axes (2D and $2 \mathrm{P}$ ) of the decagonal phase are along the $a$ and $c$ axes of the orthorhombic Taylor and Robinson phases. Both these phases have similar atomic structure [9].

\section{Taylor and Robinson phases by twinning of the RAS to the icosahedral quasicrystal}

Twinning of icosahedral quasicrystal has been studied earlier in Al-Mn [18], Al-Mn-Fe [19], Al-Cr-Si, Al-Mn-Si and Al-Mn-Cr-Si alloys [20]. A comprehensive review of twinning in quasicrystals exists [21]. A close resemblance between decagonal quasicrystal and icosahedral twins was suggested by Singh and Ranganathan [19]. The twinning was performed as a $36^{\circ}$ rotation of an icosahedron about the five-fold [ $1 \tau \tau$ 0] axis. Since there are 60 rotational symmetry operations for an icosahedral point group, the twin relationship can be expressed in 60 different ways. Earlier, Ranganathan et al. [18] explored icosahedral twinning as an explanation for the decagonal symmetry.

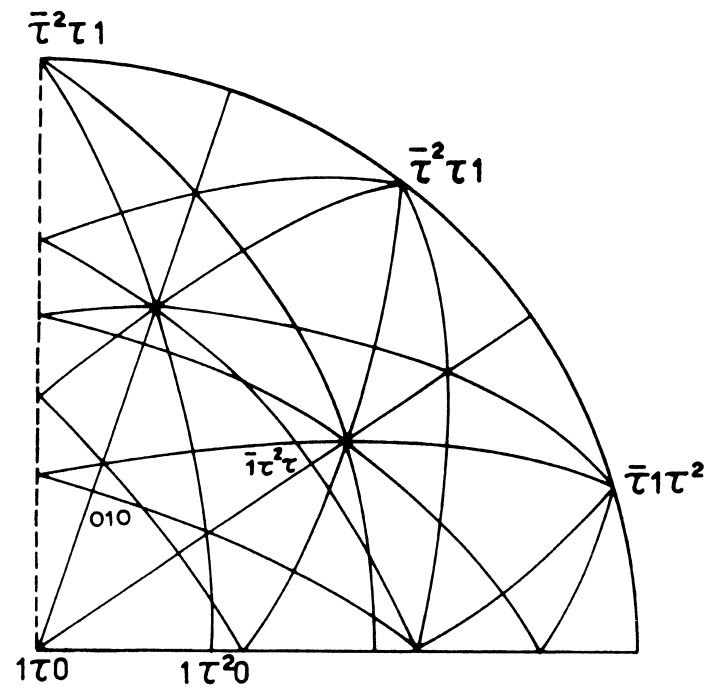

(a)

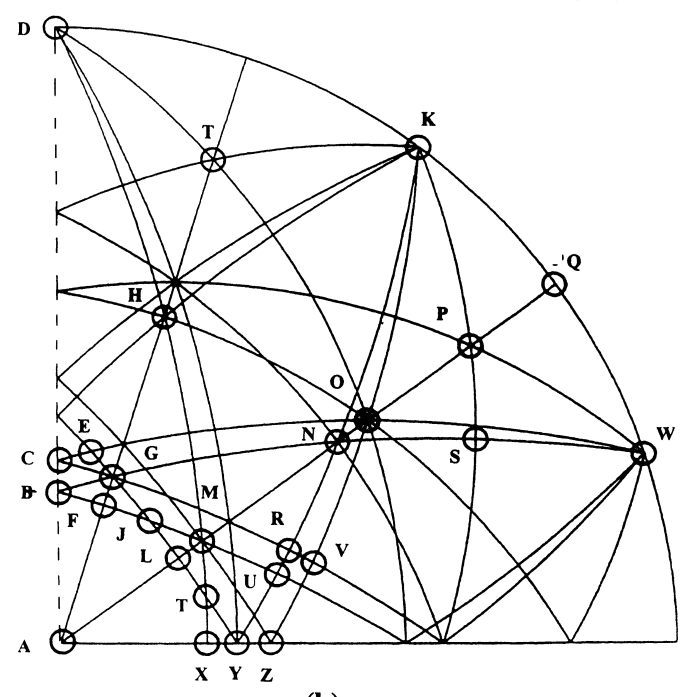

(b)

Fig. 1. Stereograms of (a) the decagonal qusicrystal generated by the twinned icosahedron model. (b) Experimental ZAPM of Al-Mn-(Pd) decagonal quasicrystal [22].

The stereogram of the decagonal quasicrystal using the $\left[\begin{array}{ll}1 & \tau\end{array}\right]$ twinned icosahedral model is shown in Fig. 1a. Fig. 1b contains the reconstructed irreducible stereographic quadrant for the $1.2 \mathrm{~nm}$ periodic Al-Mn- $(\mathrm{Pd})$ decagonal phase [22]. It can be seen from a comparison of the two stereograms that the twinned icosahedra model is able to explain the major features of the experimental stereogram (Fig. 1b). Although, some of the traces in Fig. 1a have split into two traces, as seen in Fig. 1b, the splitting is small.

The twinned icosahedra model explains the major features in the stereogram of decagonal quasicrystal. In a similar fashion, the stereogram for the approximants to the decagonal quasicrystal can be constructed using the twinning model. The Taylor approximant with similar $q / p$ ratio along the $a$ and $c$ axes, can be modeled using a twinned (1/1) Mackay type cubic approximant to the icosahedral qua- 

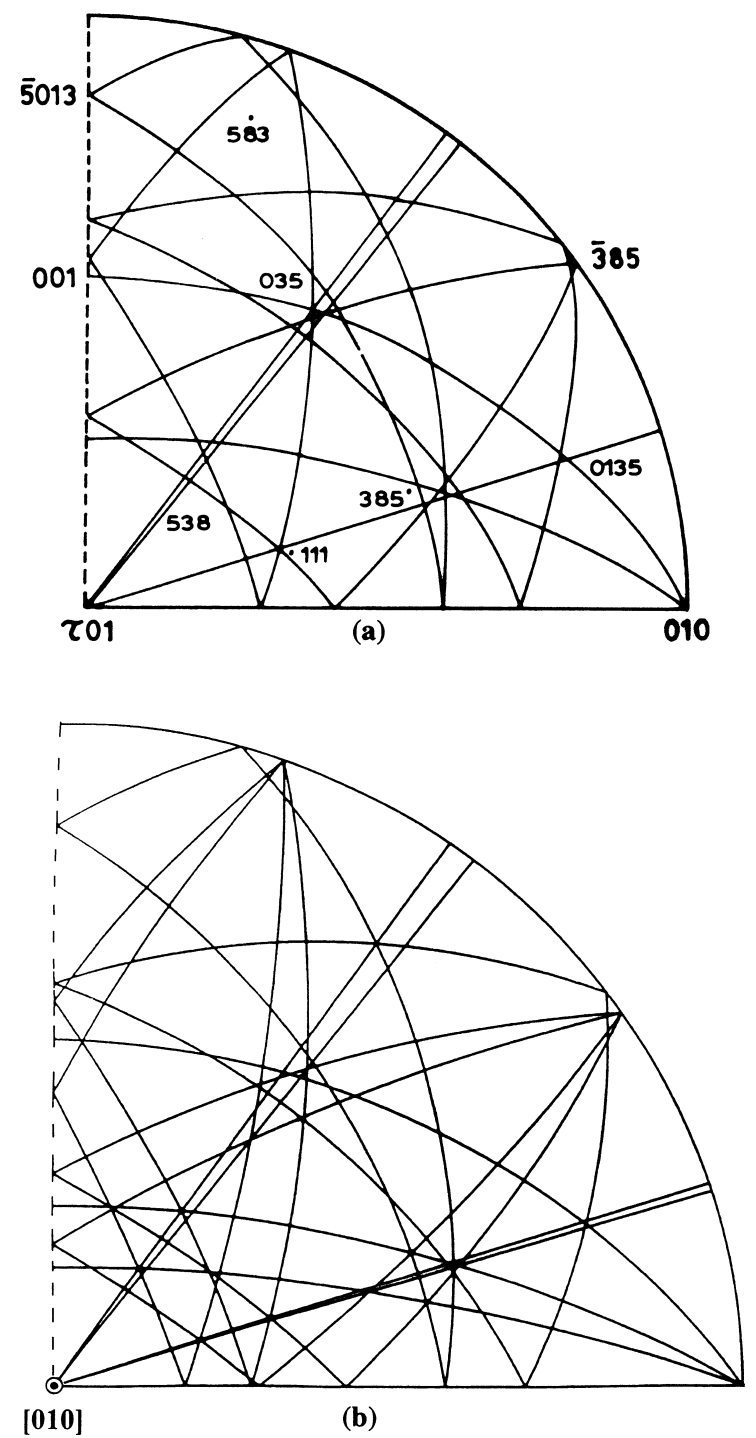

Fig. 2. Stereograms of (a) theoretical Taylor $(3 / 2,3 / 2)$ approximant (b) the experimental ZAPM of the orthorhombic $\left(\mathrm{Al}_{11} \mathrm{Mn}_{4}\right)$-Pd phase [22]

sicrystal. The twinning axis is the [ $\tau 01]$ axis of the (1/1) cubic approximant. The stereogram corresponding to this twinned model is shown in Fig. 2a. The experimental stereogram of the orthorhombic $\left(\mathrm{Al}_{11} \mathrm{Mn}_{4}\right)-\mathrm{Pd}$ phase is shown in Fig. 2b [22]. By comparing the two stereograms, it can be seen that the twinned model can explain the important features of the Taylor approximant. Some of the traces in Fig. $2 b$ have been split. This can be explained by the distortions of the icosahedra in the twinned icosahedra model. Alternately, the splitting can be understood in terms of the lowering of the symmetry of the lattice with respect to the cluster. In a similar fashion, the Robinson approximants can be derived by twinning the Little phases. The twinning of the $(1 / 1,1 / 1,3 / 2)$ about $[\tau 01]$ axis to obtain the $(2 / 1,3 / 2)$ Robinson approximant is shown in Fig. 3. Additional splitting of the traces over and above that observed for the Taylor phase is to be noted. Using this method, the series of Robin-

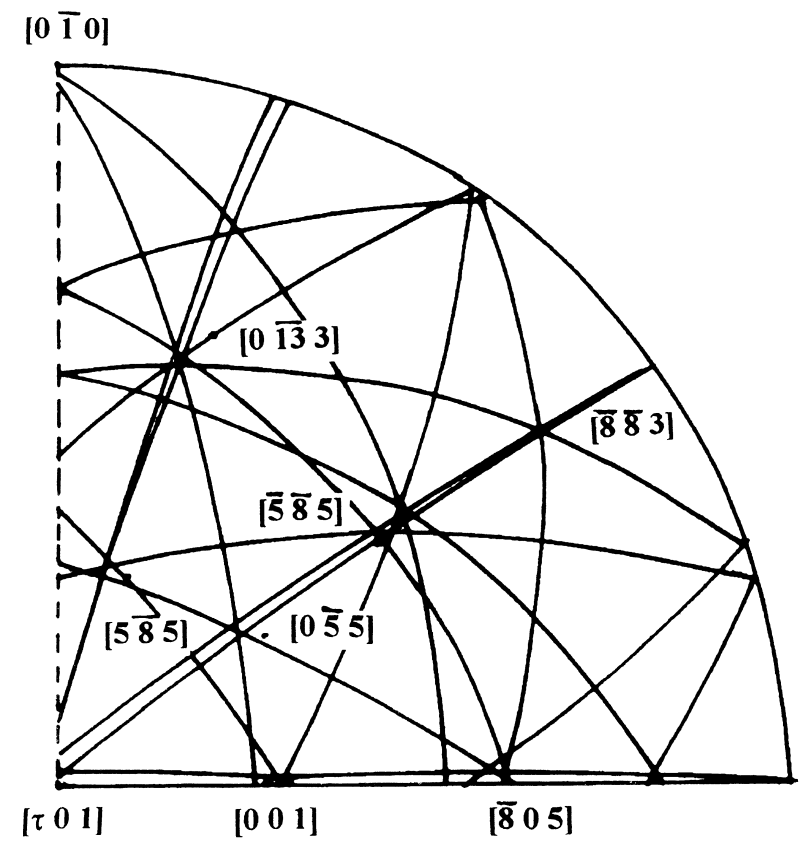

Fig. 3. Stereogram of the Robinson phase derived by the twinning of the Little approximant.

son approximants can be obtained by twinning of the Little phases with the corresponding $c / a$ ratio.

\section{Conclusions}

Clusters play an important role in the structure of quasicrystals and their rational approximants. Twinning operation leads to the various orientations of the clusters; thus, giving rise to different symmetries. Twinning of the icosahedral phase leads to the decagonal phase; twinning of the Mackay approximant leads to the Taylor approximant; whilst, the twinning of the Little approximant leads to the Robinson approximant. The apparently diverse variety of quasicrystals and their rational approximants can be brought under a unified framework by the twinning of the icosahedral quasicrystal and its rational approximants.

\section{Acknowledgements}

The authors would like to thank Dr. Alok Singh of NRIM, Tsukuba, Japan, for helpful discussions. One of the authors (K. Ramakrishnan) is grateful to the University Grants Commission, India, for providing a research fellowship. Anandh Subramaniam would like to thank CSIR for financial assistance through SRF.

\section{References}

[1] H. Zhang, K.H. Kuo, Phys. Rev. B 42 (1990) 8907-8915.

[2] M.A. Taylor, Acta Met. 8 (1960) 256-262. 
[3] M.A. Taylor, Acta Cryst. 14 (1961) 84.

[4] C. Beeli, H.U. Nissen, J. Robadey, Philos. Mag. Lett. 63 (1991) 87.

[5] X.Z. Li, K.H. Kuo, Philos. Mag. B 65 (3) (1992) 525.

[6] X.Z. Li, K.H. Kuo, Philos. Mag. B 66 (1) (1992) 117.

[7] M. Audier, D. Charre, M. deBoissieu, Philos. Mag. B 68 (1993) 607.

[8] L. Beraha, M. Duneau, M. Audier, M. Vacher, in: C. Janot, R. Mosseri (Eds.), Proceedings of the 5th International Conference on Quasicrystals, World Scientific, Singapore, 1995, p. 55.

[9] M. Audier, M. Duneau, M. Vacher, in: S. Banerjee, R.V. Ramanujam (Eds.), Advances in Physical Metallurgy, Gordon and Breach, Amsterdam, 1996, p. 61.

[10] K. Hiraga, K. Kaneko, Y. Matsuo, S. Hashimoto, Philos. Mag. B 67 (2) (1993) 193-205.

[11] G.V. Raynor, D.W. Wakeman, Proc. R. Soc. A 190 (1947) 82-101.

[12] K. Robinson, Acta Cryst. B 7 (1954) 494-497.
[13] A. Damjanovic, Acta Cryst. 14 (1961) 982-987.

[14] K. Robinson, Philos. Mag. 43 (7) (1952) 775-782.

[15] T.L. Daulton, P.C. Gibbons, K.F. Kelton, Philos. Mag. B 63 (1991) 687.

[16] A. Singh, S. Ranganathan, Mater. Sci. Eng. A 181/182 (1994) 754.

[17] X.Z. Li, D. Shi, K.H. Kuo, Philos. Mag. B 66 (1992) 331.

[18] S. Ranganathan, R. Prasad, N.K. Mukhopadhyay, Philos. Mag. Lett. 59 (6) (1989) 257-263.

[19] A. Singh, S. Ranganathan, Acta Metall. Mater. 43 (1995) 3539-3551.

[20] A.K. Srivastava, S. Ranganathan, Acta Mater. 44 (7) (1996) 2935.

[21] S. Ranganathan, A. Singh, R.K. Mandal, A. Lele, in: M.J. Yacaman, M. Torres (Eds.), Crystal-Quasicrystal Transitions, Elsevier, The Netherlands, 1993, pp. 83-88.

[22] T.L. Daulton, K.F. Kelton, Philos. Mag. B 68 (5) (1993) 697-711. 